\title{
The Impact of Price on the Profits of Fishermen Exploiting Tritrophic Prey-Predator Fish Populations
}

\author{
Meriem Bentounsi, Imane Agmour, Naceur Achtaich, and Youssef El Foutayeni \\ Analysis, Modeling and Simulation Laboratory, Hassan II University, Casablanca, Morocco \\ Correspondence should be addressed to Youssef El Foutayeni; foutayeni@gmail.com
}

Received 21 August 2017; Accepted 26 November 2017; Published 1 January 2018

Academic Editor: Abid A. Lashari

Copyright ( 2018 Meriem Bentounsi et al. This is an open access article distributed under the Creative Commons Attribution License, which permits unrestricted use, distribution, and reproduction in any medium, provided the original work is properly cited.

We define and study a tritrophic bioeconomic model of Lotka-Volterra with a prey, middle predator, and top predator populations. These fish populations are exploited by two fishermen. We study the existence and the stability of the equilibrium points by using eigenvalues analysis and Routh-Hurwitz criterion. We determine the equilibrium point that maximizes the profit of each fisherman by solving the Nash equilibrium problem. Finally, following some numerical simulations, we observe that if the price varies, then the profit behavior of each fisherman will be changed; also, we conclude that the price change mechanism improves the fishing effort of the fishermen.

\section{Introduction}

The problem of modelization is, perhaps, the most challenging in modern ecology, biology, chemistry, and many other sciences. In population dynamics, specially, in the dynamics of prey-predator marine species interactions modelization has gained a great importance. A predator is an organism that feeds another organism. The prey is the organism which the predator feeds. Predator always depends upon its prey and the predator dies if it does not get food. The first basic classic prey-predator model is renowned by Lotka-Volterra model and mathematical formulation of this model is directly related to the great work of Lotka (in 1925) and Volterra (in 1926). Thanks to this prey-predator model, other models have been proposed and studied [1-3]. In [1], the authors have considered predator-prey dynamics with predator "searching" and "handling" modes; they have derived a model that generalizes Holling's functional responses and they have proved results concerning local and global properties, including for oscillations. In [2], the authors have formulated and studied a stage-structured predator-prey model of BeddingtonDeAngelis type functional response to investigate the impact of predation over the immature prey by the juvenile predator. In [3], the authors have studied the global stability of diffusive predator-prey system of Holling-Tanner type in a bounded domain.

Let us add that many researchers have studied extended tritrophic (prey, middle predator, and top predator) models to understand the interaction of different types of species [4-6]. In [4], the dynamics of a predator-prey model with disease in super predator are investigated. In [5], the authors have studied a prey-predator model with the concept of super predator under economic perspective. In [6], the authors have made a systematic analysis of the dynamics of a predator-prey system with type II functional response, in which the predator growth rate is affected by the presence of a super predator.

In recent years, the biodiversity of marine populations is threatened by human impact, more precisely, by harvesting, which required many scientists to study bioeconomic models of fishery [ $[7,8]$. In [7], the authors have made a mathematical study of a bioeconomic model of fishing for multisite, exploited by several fishermen, except one of them which is defined as not exploitable free fishing zone. In [8], the authors proposed and analyzed an extended model for the prey-predator-scavenger in presence of harvesting to study the effects of harvesting of predator as well as scavenger.

In this paper, we have studied a tritrophic (prey, middle predator, and top predator) generalist model. The objective is 
to calculate the fishing effort that maximizes the profit of the fishermen, while respecting the conservation of the three fish populations, and also to study the effect of the variation of the price on each profit. The remaining part of this paper is organized as follows. In Section 2, we introduce the biological tritrophic model. The existence and the stability of the steady states solutions are analyzed in Section 3. The bioeconomic model of the prey, middle predator, and top predator system is proposed in Section 4. In Section 5, we compute and solve the Nash equilibrium problem. In Section 6, we solve the linear complementarity problem. In Section 7, we present some numerical simulations to show the impact of price on the profits of fishermen. Finally, a brief conclusion is given in Section 8 .

\section{Biological Model}

In this section, we consider a tritrophic prey-predator model which consists of three constituent populations, that is, prey, middle predator, and top predator. We impose that the population of prey $x(t)$ grows in the logistic manner with birth rate constant and there exist interactions between the prey and middle and top predator due to defensive ability of prey; we impose that the population of middle predator $y(t)$ grows also in the logistic manner with birth rate constant, prey $x(t)$ is favorite food for middle predator $y(t)$, and hence in the presence of favorite food the population density of middle predator $y(t)$ will increase, and there are interactions between the middle predator and top predator due to defensive ability of middle predator; in the presence of favorite food (prey and middle predator) of top predator $z(t)$ the population density of top predator $z(t)$ will increase. Hence we can write this model in mathematical terms as

$$
\begin{aligned}
& \frac{d x(t)}{d t}=r_{1} x(1-x)-\alpha x y-\beta x z, \\
& \frac{d y(t)}{d t}=r_{2} y(1-y)+\bar{\alpha} x y-\delta y z, \\
& \frac{d z(t)}{d t}=r_{3} z(1-z)+\bar{\beta} x z+\bar{\delta} y z
\end{aligned}
$$

with positive initial conditions $x(0)>0, y(0)>0, z(0)>0$.

Here $r_{1}, r_{2}$ and $r_{3}$ are the per capita growth rate of prey, middle predator, and top predator, respectively; $\alpha, \beta$, and $\delta$ are the maximum value which per capita reduction rate of $x$ and $y$ can attain, respectively; $\bar{\alpha}$ is the conversion rate of prey $x$ into middle predator $y$, and $\bar{\beta}$ and $\bar{\delta}$ are the conversion rate of prey $x$ into top predator $z$ and the conversion rate of middle predator $y$ into top predator $z$, respectively.

\section{The Steady States of the System}

Since the focus is on the growth of marine species, there is need for the steady states of the system to satisfy conditions for nonnegativity. Furthermore, it is realized that the predators cannot survive in the complete absence of their prey. System (1) has eight biologically feasible nonnegative steady states. These steady states are obtained by solving the system of equations

$$
\begin{aligned}
& r_{1}(1-x)-\alpha y-\beta z=0 \\
& r_{2}(1-y)+\bar{\alpha} x-\delta z=0 \\
& r_{3}(1-z)+\bar{\beta} x+\bar{\delta} y=0
\end{aligned}
$$

(i) $P_{1}=(0,0,0), P_{2}=(1,0,0), P_{3}=(0,1,0), P_{4}=$ $(0,0,1)$.

(ii) $P_{5}=\left(x_{5}, y_{5}, 0\right)$, where $x_{5}=r_{2}\left(r_{1}+\alpha\right) /\left(r_{1} r_{2}+\alpha \bar{\alpha}\right)$ and $y_{5}=r_{1}\left(r_{2}+\bar{\alpha}\right) /\left(r_{1} r_{2}+\alpha \bar{\alpha}\right)$.

(iii) $P_{6}=\left(x_{6}, 0, z_{6}\right)$, where $x_{6}=r_{3}\left(r_{1}-\beta\right) /\left(r_{1} r_{3}+\beta \bar{\beta}\right)$ and $z_{6}=r_{1}\left(r_{3}+\bar{\beta}\right) /\left(r_{1} r_{3}+\beta \bar{\beta}\right)$.

(iv) $P_{7}=\left(0, y_{7}, z_{7}\right)$, where $y_{7}=r_{3}\left(r_{2}-\delta\right) /\left(r_{2} r_{3}+\delta \bar{\delta}\right)$ and $z_{7}=r_{2}\left(r_{3}+\delta\right) /\left(r_{2} r_{3}+\delta \bar{\delta}\right)$.

(v) $P_{8}:=P^{*}=\left(x^{*}, y^{*}, z^{*}\right)$, where

$$
\begin{aligned}
& x^{*}=\frac{\left(r_{1} r_{2} r_{3}+r_{1} \delta \bar{\delta}+r_{3} \alpha \delta-r_{2} r_{3} \alpha-r_{2} r_{3} \beta-r_{2} \beta \bar{\delta}\right)}{\Delta}, \\
& y^{*}=\frac{\left(r_{1} r_{2} r_{3}+r_{2} \beta \bar{\beta}-r_{3} \beta \bar{\alpha}-r_{1} r_{3} \delta+r_{1} r_{3} \bar{\alpha}-r_{1} \bar{\beta} \delta\right)}{\Delta}, \\
& z^{*}=\frac{\left(r_{1} r_{2} r_{3}+r_{1} r_{2} \bar{\beta}+r_{1} r_{2} \bar{\delta}+r_{1} \bar{\alpha} \bar{\delta}-r_{2} \bar{\beta} \alpha+r_{3} \alpha \bar{\alpha}\right)}{\Delta},
\end{aligned}
$$

$$
\text { where } \Delta=r_{1} r_{2} r_{3}+r_{1} \delta \bar{\delta}+r_{2} \beta \bar{\beta}+r_{3} \alpha \bar{\alpha}-\alpha \delta \bar{\beta}+\beta \bar{\alpha} \bar{\delta} \text {. }
$$

One can see that the steady state equilibrium $P^{*}$ exists if $r_{1}>\max \{\alpha, \beta\}, r_{2}>\delta$, and $r_{3}>\max \{\delta, \bar{\beta}\}$.

3.1. Analysis of Steady States. The Jacobian matrix for system (1) is given by

$$
J=\left[\begin{array}{ccc}
J_{11} & -\alpha x & -\beta x \\
\bar{\alpha} y & J_{22} & -\delta y \\
\bar{\beta} z & \bar{\delta} z & J_{33}
\end{array}\right],
$$

where

$$
\begin{aligned}
& J_{11}=r_{1}(1-2 x)-\alpha y-\beta z, \\
& J_{22}=r_{2}(1-2 y)+\bar{\alpha} x-\delta z, \\
& J_{33}=r_{3}(1-2 z)+\bar{\beta} x+\bar{\delta} y .
\end{aligned}
$$

At any steady state solution, the Jacobian matrix is computed. Let $J_{k}=J$ denote the Jacobian evaluated at $P_{k}$ for $k=1,2,3, \ldots, 7$, the corresponding entries, and $J^{*}$ denote the Jacobian evaluated at $P^{*}$. 

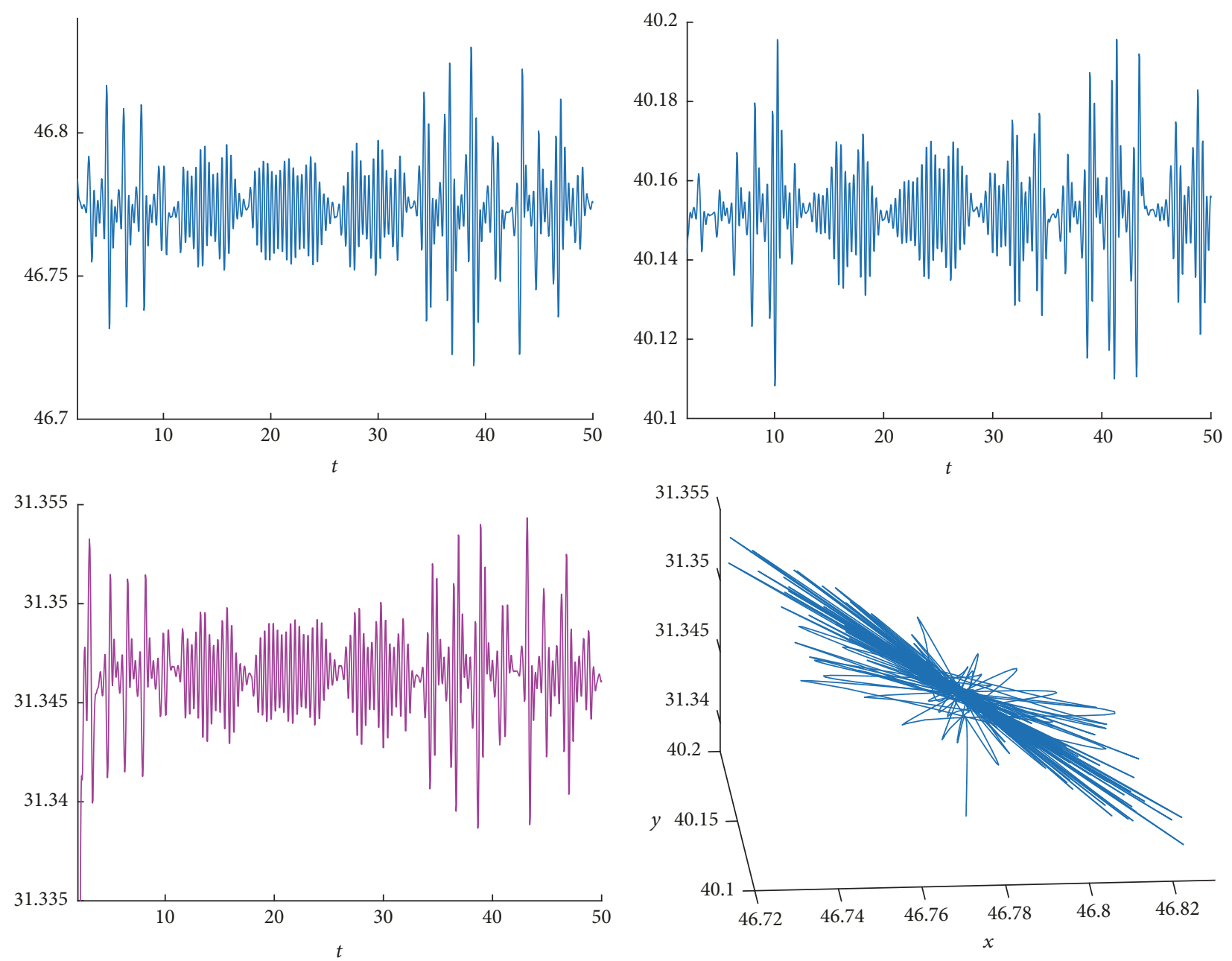

FIGURE 1: Dynamical behaviors and phase space trajectories of the three fish populations.

TABLE 1: Characteristics of the three fish populations.

\begin{tabular}{lcc}
\hline Prey & Middle predator & Top predator \\
\hline$r_{1}=5$ & $r_{2}=4$ & $r_{3}=3$ \\
$\alpha=9.10^{-6}$ & $\bar{\alpha}=8.10^{-6}$ & $\bar{\beta}=2.10^{-6}$ \\
$\beta=7.10^{-6}$ & $\delta=6.10^{-6}$ & $\bar{\delta}=10^{-6}$ \\
\hline
\end{tabular}

3.1.1. Local Stability of the Steady State $P_{1}$. For the equilibrium point $P_{1}=(0,0,0)$ the Jacobian matrix is given by

$$
J_{1}=\left[\begin{array}{ccc}
r_{1} & 0 & 0 \\
0 & r_{2} & 0 \\
0 & 0 & r_{3}
\end{array}\right] .
$$

The eigenvalues are found to be $\lambda_{1}=r_{1}>0, \lambda_{2}=r_{2}>0$, and $\lambda_{3}=r_{3}>0$, and then this point is unstable.

According to Table 1, Figure 1 shows the dynamical behaviors and phase space trajectory of the prey, middle predator, and top predator fish populations against time, beginning with the initial values $x(0)=0.01, y(0)=0.01$, and $z(0)=0.01$. By Figure 1 we find that the steady state point $P_{1}$ is unstable, and more precisely this point tends to the point $P^{*}$.

3.1.2. Local Stability of the Steady State $P_{2}$. In the same way we can consider the stationary point $P_{2}=(1,0,0)$ and find the Jacobian matrix

$$
J_{2}=\left[\begin{array}{ccc}
-r_{1} & -\alpha & -\beta \\
0 & r_{2}+\bar{\alpha} & 0 \\
0 & 0 & r_{3}+\bar{\beta}
\end{array}\right] \text {. }
$$

The eigenvalues can easily be computed, $\lambda_{1}=-r_{1}, \lambda_{2}=r_{2}+$ $\bar{\alpha}>0$, and $\lambda_{3}=r_{3}+\bar{\beta}>0$. Therefore, the point $P_{2}=(1,0,0)$ is unstable.

Following Table 1, Figure 2 shows the dynamical behaviors and phase space trajectory of the three fish populations against time, beginning with the initial values $x(0)=1.01$, and $y(0)=0.01, z(0)=0.01$. By Figure 2 we can see that the 

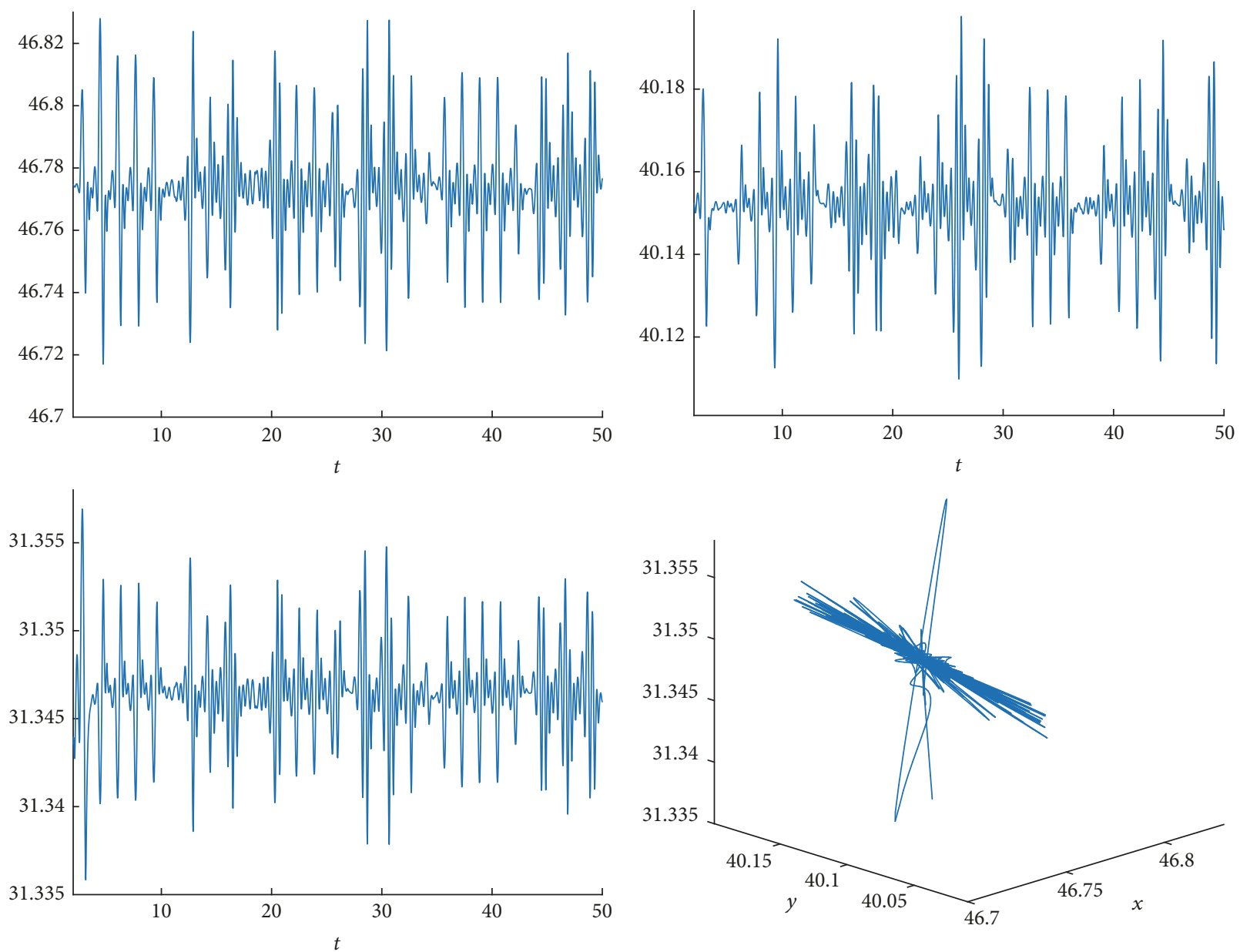

FIGURE 2: Dynamical behaviors and phase space trajectories of the three fish populations.

steady state point $P_{2}$ is unstable, and more precisely this point tends to the point $P^{*}$ too.

3.1.3. Local Stability of the Steady State $P_{3}$. For the point $P_{3}=$ $(0,1,0)$ we have the Jacobian matrix $J_{3}$ which is written in the form

$$
J_{3}=\left[\begin{array}{ccc}
r_{1}-\alpha & 0 & 0 \\
\bar{\alpha} & -r_{2} & -\delta \\
0 & 0 & r_{3}+\bar{\delta}
\end{array}\right]
$$

The eigenvalues are $\lambda_{1}=r_{1}-\alpha, \lambda_{2}=-r_{2}$, and $\lambda_{3}=r_{3}+\bar{\delta}>0$. Then, the point $P_{3}=(0,1,0)$ is unstable.

According to Table 1, Figure 3 shows the dynamical behaviors and phase space trajectory of the three fish populations against time, beginning with the initial values $x(0)=$ $0.01, y(0)=1.01$, and $z(0)=0.01$. By Figure 3 we can see that the steady state point $P_{3}$ is also unstable and tends to the point $P^{*}$.

3.1.4. Local Stability of the Steady State $P_{4} . P_{4}=(0,0,1)$ is stable if $r_{1}-\beta<0$ and $r_{2}-\delta<0$; if not, it is unstable.
In fact, the Jacobian matrix of the system in this state is written as

$$
J_{4}=\left[\begin{array}{ccc}
r_{1}-\beta & 0 & 0 \\
0 & r_{2}-\delta & 0 \\
\bar{\beta} & \bar{\delta} & -r_{3}
\end{array}\right] .
$$

The eigenvalues are $\lambda_{1}=r_{1}-\beta, \lambda_{2}=r_{2}-\delta$, and $\lambda_{3}=-r_{3}$. Therefore, if $r_{1}-\beta<0$ and $r_{2}-\delta<0$, then the point $P_{4}=$ $(0,0,1)$ is stable; if not, it is an unstable point.

For the same values parameters in Table 1, Figure 4 indicates the dynamical behaviors and phase space trajectory of the three fish populations against time, beginning with the initial values $x(0)=0.01, y(0)=0.01$, and $z(0)=1.01$. Following Figure 4 we can see that the steady state point $P_{3}$ is unstable and also tends to the point $P^{*}$.

3.1.5. Local Stability of the Steady State $P_{5}$. For the equilibrium point $P_{5}=\left(x_{5}, y_{5}, 0\right)$ the Jacobian matrix is given by

$$
J_{5}=\left[\begin{array}{ccc}
-r_{1} x_{5} & -\alpha x_{5} & -\beta x_{5} \\
\bar{\alpha} y_{5} & -r_{2} y_{5} & -\delta y_{5} \\
0 & 0 & r_{3}+\bar{\beta} x_{5}+\bar{\delta} y_{5}
\end{array}\right] .
$$



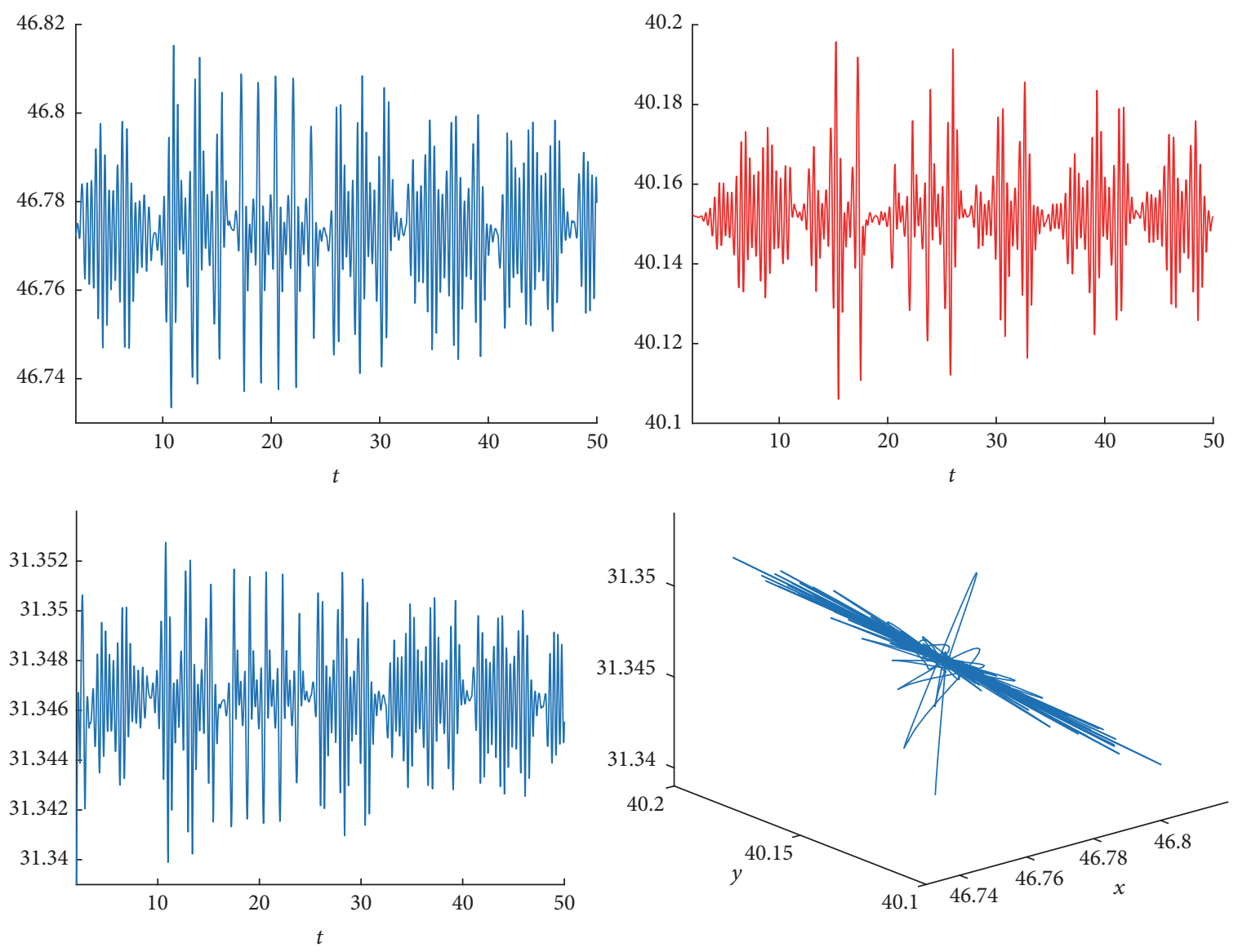

FIgURE 3: Dynamical behaviors and phase space trajectories of the three fish populations.

The eigenvalues are

$$
\begin{aligned}
& \lambda_{1} \\
& \quad=-\frac{1}{2}\left(r_{1} x_{5}+r_{2} y_{5}+\sqrt{\left(r_{1} x_{5}-r_{2} y_{5}\right)^{2}-4 \alpha \bar{\alpha} x_{5} y_{5}}\right), \\
& \lambda_{2} \\
& \quad=-\frac{1}{2}\left(r_{1} x_{5}+r_{2} y_{5}-\sqrt{\left(r_{1} x_{5}-r_{2} y_{5}\right)^{2}-4 \alpha \bar{\alpha} x_{5} y_{5}}\right), \\
& \lambda_{3}=r_{3}+\bar{\beta} x_{5}+\bar{\delta} y_{5} .
\end{aligned}
$$

We have $\lambda_{3}>0$; then, the point $P_{5}=\left(x_{5}, y_{5}, 0\right)$ is unstable.

Following Table 1, Figure 5 represents the dynamical behaviors and phase space trajectory of the three fish populations against time, beginning with the initial values $x(0)=$ $1.01, y(0)=1.02$, and $z(0)=0.01$. Following Figure 5 we can deduce that the steady state point $P_{5}$ is unstable and also tends to the point $P^{*}$.

3.1.6. Local Stability of the Steady State $P_{6}$. For the equilibrium point $P_{6}=\left(x_{6}, 0, z_{6}\right)$ the Jacobian matrix is given by

$$
J_{6}=\left[\begin{array}{ccc}
-r_{1} x_{6} & -\alpha x_{6} & -\beta x_{6} \\
0 & r_{2}+\bar{\alpha} x_{6}-\delta z_{6} & 0 \\
\bar{\beta} z_{6} & \bar{\delta} z_{6} & r_{3} z_{6}
\end{array}\right]
$$

the eigenvalues are

$\lambda_{1}$

$$
=-\frac{1}{2}\left(r_{1} x_{6}+r_{3} z_{6}-\sqrt{\left(r_{1} x_{6}-r_{3} z_{6}\right)^{2}-4 \beta \bar{\beta} x_{6} z_{6}}\right),
$$

$\lambda_{2}$

$$
=-\frac{1}{2}\left(r_{1} x_{6}+r_{3} z_{6}+\sqrt{\left(r_{1} x_{6}-r_{3} z_{6}\right)^{2}-4 \beta \bar{\beta} x_{6} z_{6}}\right),
$$

$$
\lambda_{3}=r_{2}+\bar{\alpha} x_{6}-\delta z_{6}
$$

We have $\lambda_{1}>0$ and $\lambda_{2}>0$. Therefore, the point $P_{6}=$ $\left(x_{6}, 0, z_{6}\right)$ is unstable.

According to Table 1, Figure 6 shows the dynamical behaviors and phase space trajectory of the three marine species against time, beginning with the initial values $x(0)=$ $1.05, y(0)=0.01$, and $z(0)=1.5$. Following Figure 6 we can deduce that the steady state point $P_{6}$ is unstable and also tends to the point $P^{*}$. 

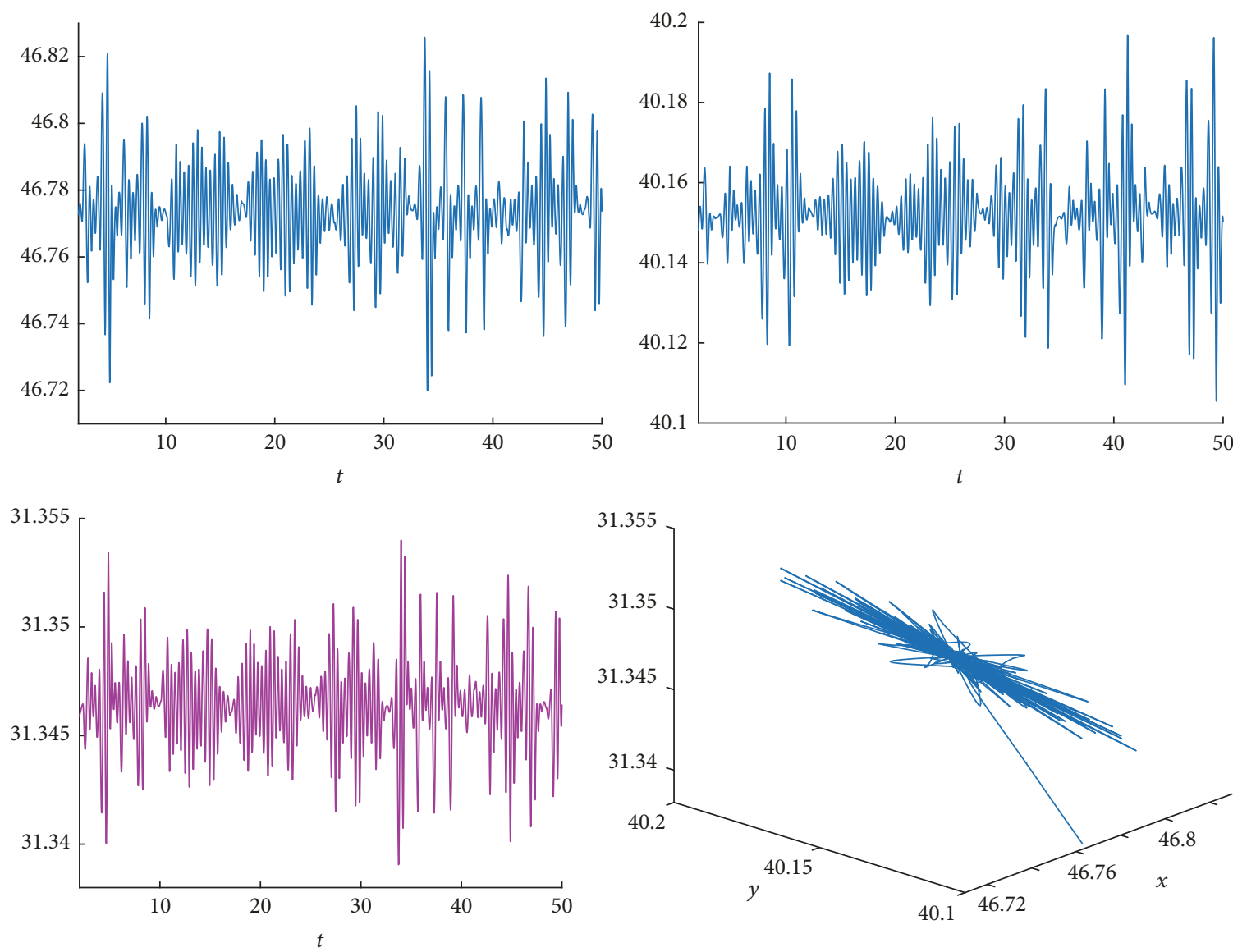

FIgURE 4: Dynamical behaviors and phase space trajectories of the three fish populations.

3.1.7. Local Stability of the Steady State $P_{7}$. For the equilibrium point $P_{7}=\left(0, y_{7}, z_{7}\right)$ the Jacobian matrix is given by

$$
J_{7}=\left[\begin{array}{ccc}
r_{1}-\alpha y_{7}-\beta z_{7} & 0 & 0 \\
\bar{\alpha} y_{7} & -r_{2} y_{7} & -\delta y_{7} \\
\bar{\beta} z_{7} & \bar{\delta} z_{7} & -r_{3} z_{7}
\end{array}\right] .
$$

the eigenvalues are

$$
\begin{aligned}
\lambda_{1} & \\
& =-\frac{1}{2}\left(r_{2} y_{7}+r_{3} z_{7}-\sqrt{\left(r_{2} y_{7}+r_{3} z_{7}\right)^{2}-4 \delta \bar{\delta} y_{7} z_{7}}\right), \\
\lambda_{2} & \\
& =-\frac{1}{2}\left(r_{2} y_{7}+r_{3} z_{7}+\sqrt{\left(r_{2} y_{7}+r_{3} z_{7}\right)^{2}-4 \delta \bar{\delta} y_{7} z_{7}}\right), \\
\lambda_{3} & =r_{1}-\alpha y_{7}-\beta z_{7} .
\end{aligned}
$$

We have $\lambda_{1}>0$ and $\lambda_{2}>0$. Therefore, the point $P_{7}=$ $\left(0, y_{7}, z_{7}\right)$ is unstable.

Following Table 1, Figure 7 shows the dynamical behaviors and phase space trajectory of the three marine species against time, beginning with the initial values $x(0)=0.01$, $y(0)=1.4$, and $z(0)=1.65$. By Figure 7 we can conclude that the steady state point $P_{7}$ is unstable and also tends to the point $P^{*}$.

3.1.8. Local Stability of the Steady State $P^{*}$. As usual, one can consider the corresponding linearized system (the Jacobian matrix) and determine the characteristic equation for the eigenvalues. The Jacobian matrix (in the equilibrium point $P^{*}$ ) reads

$$
J^{*}=\left[\begin{array}{ccc}
-r_{1} x^{*} & -\alpha x^{*} & -\beta x^{*} \\
\bar{\alpha} y^{*} & -r_{2} y^{*} & -\delta y^{*} \\
\bar{\beta} z^{*} & \bar{\delta} z^{*} & -r_{3} z^{*}
\end{array}\right]
$$

resulting in the characteristic equation and trying to apply Routh-Hurwitz conditions. We find

$$
P(\lambda)=a_{0} \lambda^{3}+a_{1} \lambda^{2}+a_{2} \lambda+a_{3}
$$

where

$$
\begin{aligned}
a_{0} & =1, \\
a_{1} & =r_{1} x^{*}+r_{2} y^{*}+r_{3} z^{*} \\
a_{2} & =r_{3} z^{*}\left(r_{1} x^{*}+r_{2} y^{*}\right)+x^{*} y^{*}\left(\alpha \bar{\alpha}+r_{1} r_{2}\right) \\
& +x^{*} z^{*} \beta \bar{\beta}+y^{*} z^{*} \delta \bar{\delta}
\end{aligned}
$$



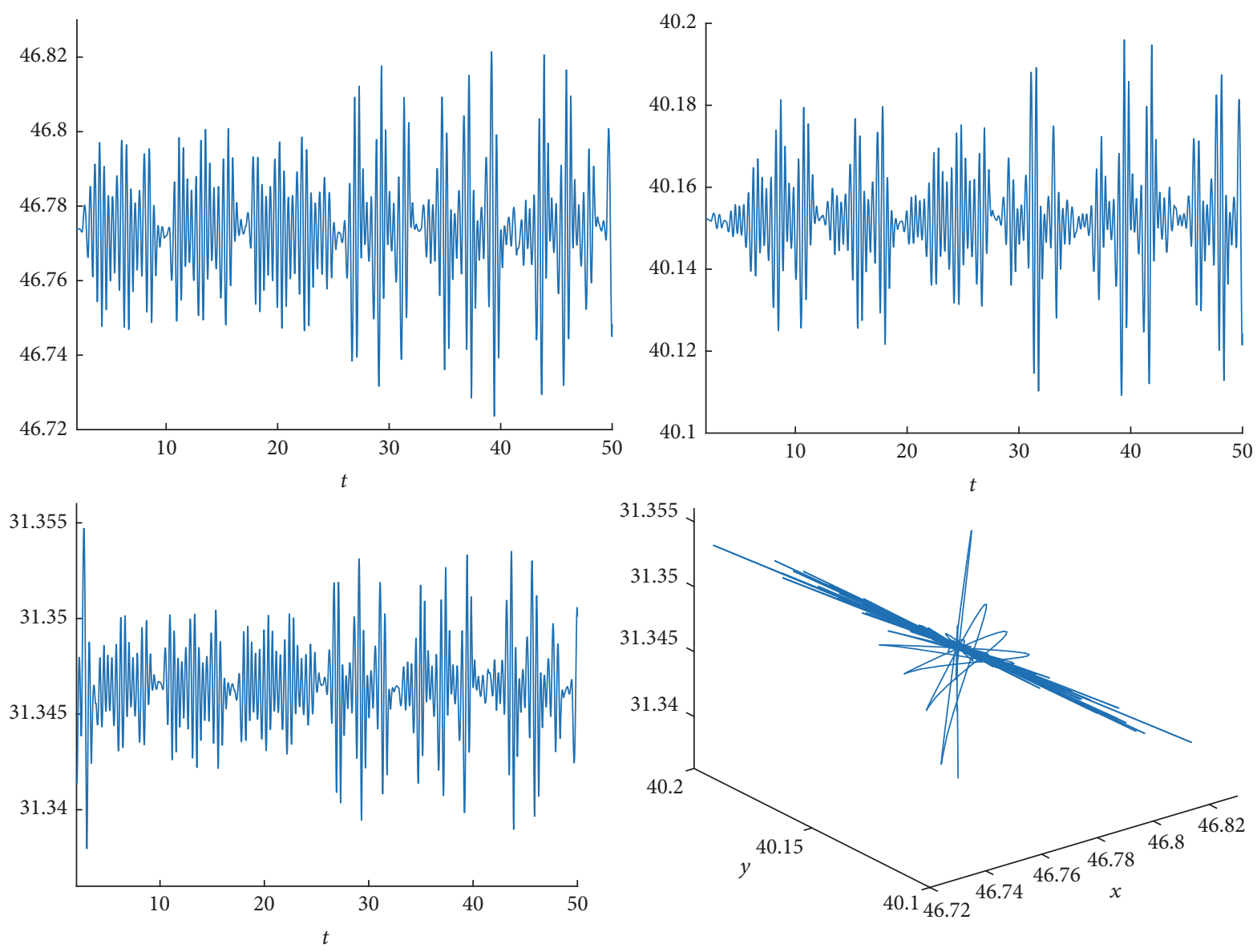

FIGURE 5: Dynamical behaviors and phase space trajectories of the three fish populations.

$$
\begin{aligned}
a_{3} & =z^{*}\left(r_{1} x^{*}+r_{2} y^{*}\right)\left(x^{*} \beta \bar{\beta}+y^{*} \delta \bar{\delta}\right) \\
& -\bar{\delta} y^{*} z^{*}\left(\delta r_{2} y-\bar{\alpha} \beta x^{*}\right) \\
& -\bar{\beta} x^{*} z^{*}\left(\beta r_{1} x^{*}+\alpha \delta y^{*}\right)+r_{3} x^{*} y^{*} z^{*}\left(\alpha \bar{\alpha}+r_{1} r_{2}\right) .
\end{aligned}
$$

We can easily verify that $a_{0}, a_{1}, a_{2}, a_{3}$, and $a_{1} a_{2}-a_{0} a_{3}$ are all positive. Thus, the Routh-Hurwitz conditions are satisfied. Therefore, the point $P^{*}=\left(x^{*}, y^{*}, z^{*}\right)$ is locally asymptotically stable.

For the values parameters quoted in Table 1, Figure 8 shows the dynamical behaviors and phase space trajectory of the three marine species against time, beginning with the initial values $x(0)=46.7, y(0)=39.9$, and $z(0)=31.1$. By Figure 8 one can see that the steady state point $P^{*}$ is locally asymptotically stable.

More precisely, beginning with different initial values we can note that the three fish populations tend to the point $P^{*}$.

\section{Bioeconomic Model}

The main purpose of this section is to define and study a bioeconomic model for two fishermen who catch the three fish populations. The model for the evolution of these three fish populations becomes

$$
\begin{aligned}
& \frac{d x(t)}{d t}=r_{1} x(1-x)-\alpha x y-\beta x z-q_{1} E_{1} x, \\
& \frac{d y(t)}{d t}=r_{2} y(1-y)+\bar{\alpha} x y-\delta y z-q_{2} E_{2} y, \\
& \frac{d z(t)}{d t}=r_{3} z(1-z)+\bar{\beta} x z+\bar{\delta} y z-q_{3} E_{3} z .
\end{aligned}
$$

The capturability coefficient $q$ is a key parameter in the validation process of the fishing simulation model, which is assumed to be constant. Fishing effort is defined as the product of fishing activity and fishing power. The fishing effort deployed by a fleet is the sum of these products on all fishing units in the fleet, while fishing power is the ability of a fishing unit to catch fish. However, it is interesting to note that, according to the literature, effort depends on several variables, for example, ship, number of hours spent fishing, number of days spent fishing, number of stolen sorties, technology, fishing equipment, and crew.

However, in this paper, effort is treated as a variable that combines all of these factors. 

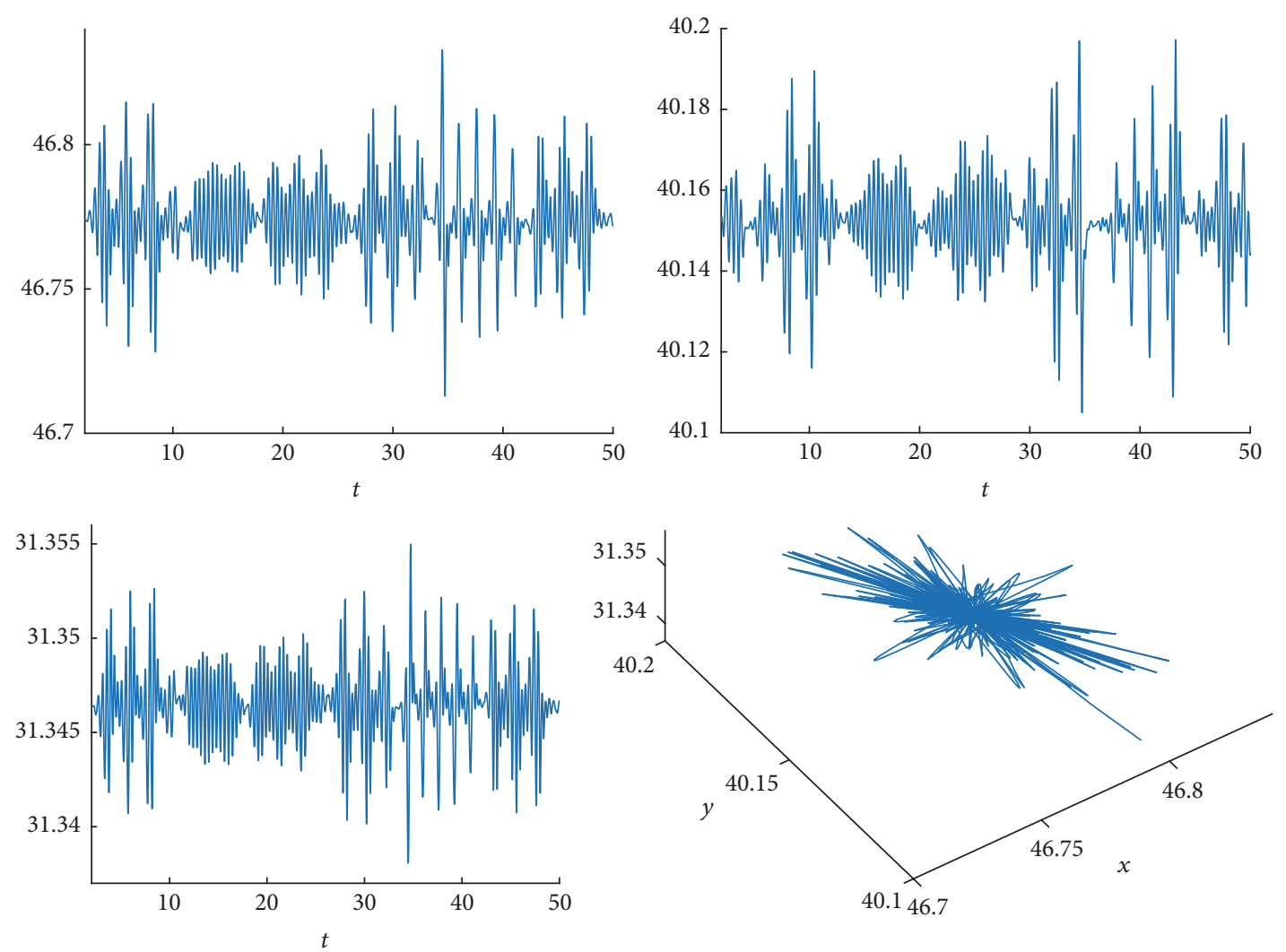

FIgURE 6: Dynamical behaviors and phase space trajectories of the three marine species.

The expression of biomass as a function of fishing effort at biological equilibrium is the solution of the system

$$
\begin{aligned}
& r_{1}(1-x)=\alpha y+\beta z+q_{1} E_{1}, \\
& r_{2}(1-y)=-\bar{\alpha} x+\delta z+q_{2} E_{2}, \\
& r_{3}(1-z)=-\bar{\beta} x-\bar{\delta} y+q_{3} E_{3} .
\end{aligned}
$$

This solution of this system (20) is given by

$$
\begin{aligned}
& x=a_{11} E_{1}+a_{12} E_{2}+a_{13} E_{3}+x^{*}, \\
& y=a_{21} E_{1}+a_{22} E_{2}+a_{23} E_{3}+y^{*}, \\
& z=a_{31} E_{1}+a_{32} E_{2}+a_{33} E_{3}+z^{*},
\end{aligned}
$$

where

$$
\begin{aligned}
& a_{11}=\frac{-\left(\delta \bar{\delta} q_{1}-r_{2} r_{3} q_{1}\right)}{\Delta}, \\
& a_{12}=\frac{\left(\beta \bar{\delta} q_{2}+\alpha r_{3} q_{2}\right)}{\Delta}, \\
& a_{13}=\frac{\left(-\delta \alpha q_{3}+\beta r_{2} q_{3}\right)}{\Delta},
\end{aligned}
$$

$$
\begin{aligned}
& a_{21}=\frac{\left(\delta \bar{\beta} q_{1}-\bar{\alpha} r_{3} q_{1}\right)}{\Delta}, \\
& a_{22}=\frac{\left(-\beta \bar{\beta} q_{2}-r_{1} r_{3} q_{2}\right)}{\Delta}, \\
& a_{23}=\frac{\left(\beta \bar{\alpha} q_{3}+\delta r_{1} q_{3}\right)}{\Delta}, \\
& a_{31}=\frac{\left(-\bar{\alpha} \bar{\delta} q_{1}-\bar{\beta} r_{2} q_{1}\right)}{\Delta}, \\
& a_{32}=\frac{\left(\alpha \bar{\beta} q_{2}-\bar{\delta} r_{1} q_{2}\right)}{\Delta}, \\
& a_{33}=\frac{\left(-\alpha \bar{\alpha} q_{3}-r_{1} r_{2} q_{3}\right)}{\Delta} .
\end{aligned}
$$

In matrix form, this solution can be written as $X=-A E+X^{*}$, where $A=\left(-a_{i j}\right)_{1 \leq i, j \leq 3}$ and $X^{*}=\left(x^{*}, y^{*}, z^{*}\right)^{T}$ with $a_{i i}<0$ for all $i=1,2,3$.

4.1. The Net Economic Revenue. Simultaneously, an algebraic equation is also included due to the consideration of the economic profit of harvesting. According to Gordon's economic theory

(i) $\Pi_{i}(E)=$ Total Revenue $(\mathrm{TR})_{i}-$ Total Cost $(\mathrm{TC})_{i}$, 

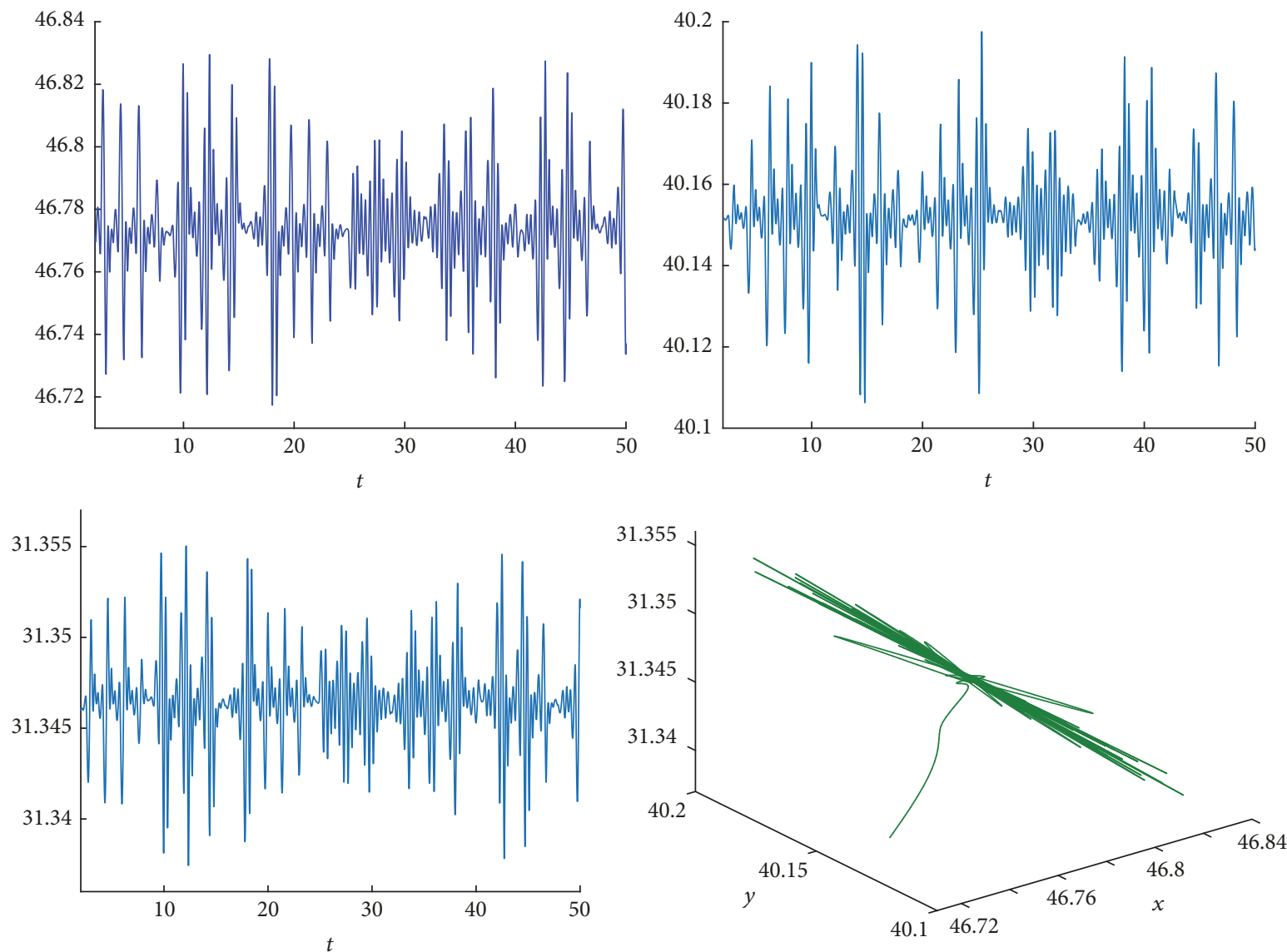

FIGURE 7: Dynamical behaviors and phase space trajectories of the three fish populations.

where the total revenue $(\mathrm{TR})_{i}$ and total cost $(\mathrm{TC})_{i}$ are given by

(a) $(\mathrm{TR})_{i}=$ Price $p_{i} \times$ Catches $H_{i j}$.

We note that $H_{i j}=q_{j} E_{i j} X_{j}$ represent the catches of species $j$ by the fisherman $i\left(X_{1}=x, X_{2}=y\right.$, and $\left.X_{3}=z\right)$, and $E_{i j}$ is the effort of the fisherman $i$ to exploit the species $j$. It is clear that $H_{j}=\sum_{i=1}^{2} H_{i j}$ is the total catches of species $j$ by all fishermen.

According to the above notations we have

$$
\begin{aligned}
(\mathrm{TR})_{i} & =p_{1} H_{i 1}+p_{2} H_{i 2}+p_{3} H_{i 3} \\
& =\left\langle E^{i},-p q A E^{i}+p q X^{*}-p q A E^{j}\right\rangle .
\end{aligned}
$$

(b) $(\mathrm{TC})_{i}=\left\langle c^{i}, E^{i}\right\rangle$,

where $c^{i}$ is a constant cost per unit of harvesting effort of the fisherman $i$.

The profit for each fisherman is represented by the function $\Pi_{i}(E)$, so that the profit of fisherman $i$ is given by

$$
\Pi_{i}(E)=\left\langle E^{i},-p q A E^{i}-p q X^{*}-c^{i}-p q A E^{j}\right\rangle .
$$

To maintain the biodiversity of the three fish populations, it is natural to assume that all biomasses remain positive; therefore $X=-A E+X^{*} \geq 0$. In other words, for the fisherman $i$ we must have $A E^{i} \leq A E^{j}-X^{*}$.

\section{Nash Equilibrium}

Each of the two fishermen tries to maximize their profits and reach a fishing effort that is an optimal response to the effort of the other fisherman. Therefore, we have a Nash equilibrium situation where each fisherman's strategy is optimal, taking into account the strategy of the second fisherman. This problem can be translated into the two following mathematical problems.

The first fisherman must solve this problem $\left(p_{1}\right)$ :

$$
\begin{aligned}
\max & \prod\left(E^{1}\right) \\
& =\left\langle E^{1},-p q A E^{1}+p q X^{*}-c^{1}-p q A E^{2}\right\rangle \\
\text { subject to } & A E^{1} \leq-A E^{2}+X^{*} \\
& E^{1} \geq 0 \\
& E^{2} \text { given. }
\end{aligned}
$$



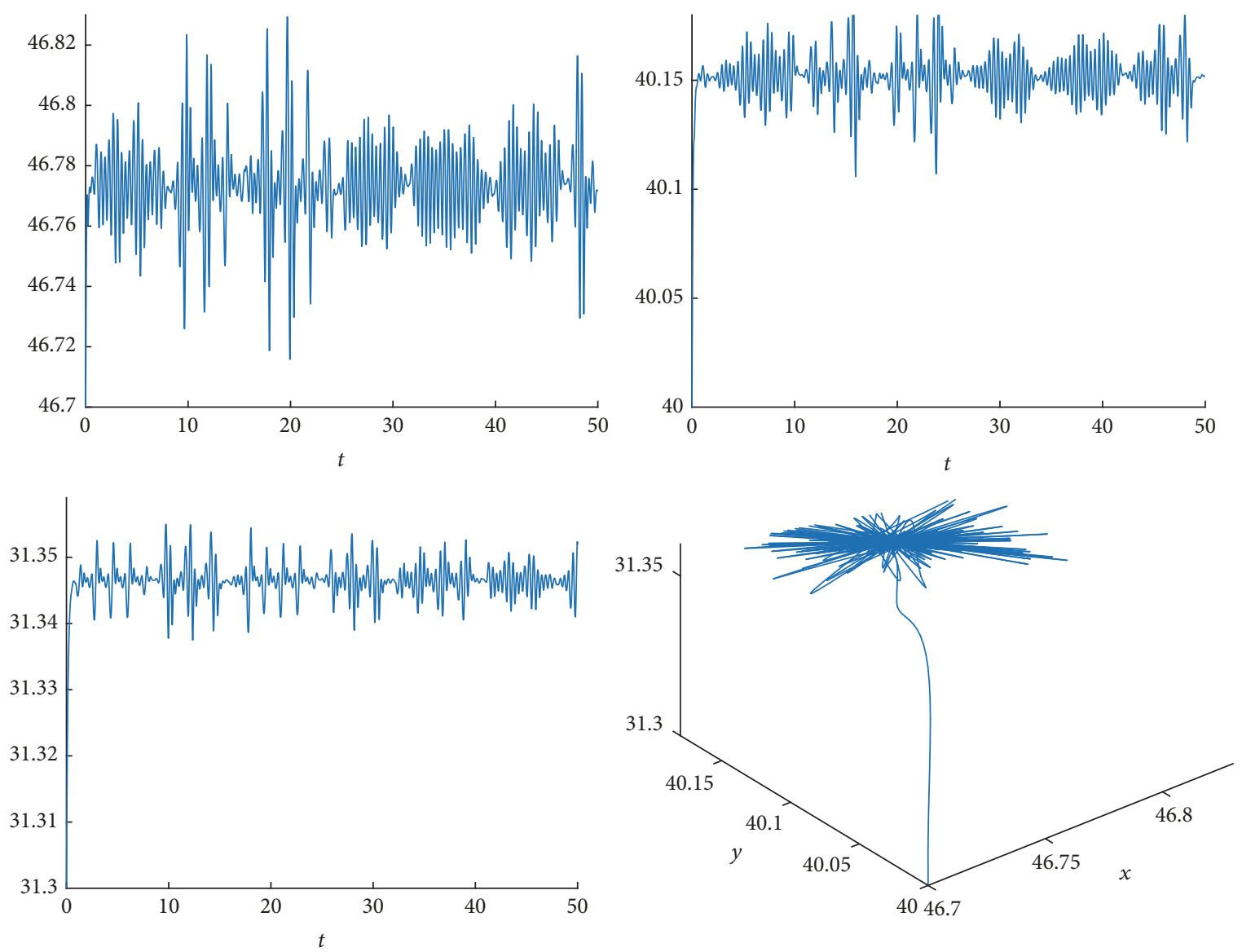

FIGURE 8: Dynamical behaviors and phase space trajectories of the three fish populations.

The second fisherman must solve this problem $\left(p_{2}\right)$ :

$$
\begin{aligned}
\max & \prod\left(E^{2}\right) \\
& =\left\langle E^{2},-p q A E^{2}+p q X^{*}-c^{2}-p q A E^{1}\right\rangle
\end{aligned}
$$

subject to $A E^{2} \leq-A E^{1}+X^{*}$

$$
\begin{aligned}
& E^{2} \geq 0 \\
& E^{1} \text { given. }
\end{aligned}
$$

By definition, the point $\left(E_{1}, E_{2}\right)$ is called Nash equilibrium point if and only if $E_{1}$ is a solution of problem $\left(p_{1}\right)$ for given $E_{2}$, and $E_{2}$ is a solution of problem $\left(p_{2}\right)$ for given $E_{1}$.

By applying the essential conditions of Karush-KuhnTucker to the problem $\left(p_{1}\right)$ we will ensure the existence of constants $u^{1} \in \mathbb{R}_{+}^{3}, v^{1} \in \mathbb{R}_{+}^{3}$, and $\lambda^{1} \in \mathbb{R}_{+}^{3}$ such that

$$
\begin{aligned}
& 2 p q A E^{1}+c^{1}-p q X^{*}+p q A E^{2}-u^{1}+A^{T} \lambda^{1}=0, \\
& A E^{1}+v^{1}=A E^{2}+X^{*}, \\
& \left\langle u^{1}, E^{1}\right\rangle=\left\langle\lambda^{1}, v^{1}\right\rangle=0 .
\end{aligned}
$$

In the same way, by applying the essential conditions of Karush-Kuhn-Tucker to the problem $\left(p_{2}\right)$ we will ensure the existence of constants $u^{2} \in \mathbb{R}_{+}^{3}, v^{2} \in \mathbb{R}_{+}^{3}$, and $\lambda^{2} \in \mathbb{R}_{+}^{3}$ such that

$$
\begin{aligned}
& 2 p q A E^{2}+c^{2}-p q X^{*}+p q A E^{1}-u^{2}+A^{T} \lambda^{2}=0, \\
& A E^{2}+v^{2}=A E^{1}+X^{*}, \\
& \left\langle u^{2}, E^{2}\right\rangle=\left\langle\lambda^{2}, v^{2}\right\rangle=0 .
\end{aligned}
$$

We can notice that the two preceding problems can be written in the form of a single problem which is the following:

$$
\begin{aligned}
& u^{1}= 2 p q A E^{1}+c^{1}-p q X^{*}+p q A E^{2} \\
&+A^{T} \lambda^{1}, \\
& u^{2}= 2 p q A E^{2}+c^{2}-p q X^{*}+p q A E^{1} \\
&+A^{T} \lambda^{2}, \\
& v^{1}=-A E^{1}-A E^{2}+X^{*}, \\
& v^{2}=-A E^{2}-A E^{1}+X^{*}, \\
&\left\langle u^{i}, E^{i}\right\rangle=\left\langle\lambda^{i}, v^{i}\right\rangle=0 \quad \forall i=1,2, \\
& u^{i}, E^{i}, \lambda^{i}, v^{i} \geq 0 \quad \forall i=1,2 .
\end{aligned}
$$


To maintain the biodiversity of fish populations, it is natural to assume that all biomasses remain strictly positive; that is, $X^{*}>0$; therefore $v^{1}=v^{2}>0$. As the scalar product of $\left(\lambda^{i}\right)_{i=1,2}$ and $\left(v^{i}\right)_{i=1,2}$ is zero, $\lambda^{i}=0$ for all $i=1,2$. In what follows in this paper, we denote $v:=v^{1}=v^{2}$. So we have the following expressions:

$$
\begin{aligned}
u^{1} & =2 p q A E^{1}+p q A E^{2}+c^{1}-p q X^{*}, \\
u^{2} & =2 p q A E^{2}+p q A E^{1}+c^{2}-p q X^{*}, \\
v & =-A E^{1}-A E^{2}+X^{*}, \\
\left\langle u^{i}, E^{i}\right\rangle & =\left\langle\lambda^{i}, v^{i}\right\rangle=0 \quad \forall i=1,2, \\
u^{i}, E^{i}, \lambda^{i}, v^{i} & \geq 0 \quad \forall i=1,2 ;
\end{aligned}
$$

thus,

$$
\begin{aligned}
\left(\begin{array}{c}
u^{1} \\
u^{2} \\
v
\end{array}\right)= & \left(\begin{array}{ccc}
2 p q A & p q A & A^{T} \\
p q A & 2 p q A & 1 \\
-A & -A & 1
\end{array}\right)\left(\begin{array}{c}
E^{1} \\
E^{2} \\
0
\end{array}\right) \\
& +\left(\begin{array}{c}
c^{1}-p q X^{*} \\
c^{2}-p q X^{*} \\
X^{*}
\end{array}\right) .
\end{aligned}
$$

Let us denote

$$
\begin{aligned}
z & =\left(\begin{array}{c}
E^{1} \\
E^{2} \\
0
\end{array}\right), \\
w & =\left(\begin{array}{c}
u^{1} \\
u^{2} \\
v
\end{array}\right), \\
M & =\left(\begin{array}{ccc}
2 p q A & p q A & A^{T} \\
p q A & 2 p q A & 1 \\
-A & -A & 1
\end{array}\right], \\
b & =\left(\begin{array}{c}
c-p q X^{*} \\
c-p q X^{*} \\
X^{*}
\end{array}\right) .
\end{aligned}
$$

\section{Linear Complementarity Problem}

The Nash equilibrium problem is equivalent to the following linear complementarity problem $\operatorname{LCP}(M, b)$.

Find vectors $z, w \in \mathbb{R}^{9}$ such that $w=M z+b \geq 0, z, w \geq 0$, and $z^{T} w=0$.

Using the following theorem we can prove that this linear complementarity problem $\operatorname{LCP}(M, b)$ has a unique solution.

Theorem 1. $\operatorname{LCP}(M, b)$ has a unique solution for every $b$ if and only if $M$ is a $P$-matrix.
Proof. We have $a_{i i}<0$ for all $i=1,2,3$ and $\Delta>0$ so if we note by $\left(M_{i}\right)_{i=1, \ldots, 9}$ the submatrix of $M$, we obtain

$$
\begin{aligned}
& \operatorname{det}\left(M_{1}\right)=-2 p_{01} q_{1} a_{11}>0, \\
& \operatorname{det}\left(M_{2}\right)=4 p_{1} q_{1} p_{2} q_{2} q_{2} q_{1} r_{3} q_{2} K_{2} \Delta>0, \\
& \operatorname{det}\left(M_{3}\right)=8 p_{1} q_{1}^{2} p_{2} q_{2}^{2} p_{3} q_{3}^{2} \Delta^{2}>0, \\
& \operatorname{det}\left(M_{4}\right)=-12 a_{11} p_{1}^{2} q_{1}^{3} p_{2} q_{2}^{2} p_{3} q_{3}^{2} \Delta^{2}>0, \\
& \operatorname{det}\left(M_{5}\right)=18 p_{1}^{2} q_{1}^{4} p_{2}^{2} q_{2}^{4} p_{3} r_{3} q_{3}^{2} \Delta^{3}>0, \\
& \operatorname{det}\left(M_{6}\right)=27 p_{1}^{2} q_{1}^{4} p_{2}^{2} q_{2}^{4} p_{3}^{2} q_{3}^{4} \Delta^{4}>0, \\
& \operatorname{det}\left(M_{7}\right)=-9 p_{1} q_{1}^{3} p_{2}^{2} q_{2}^{4} p_{3}^{2} q_{3}^{4} a_{11} \Delta^{4}>0, \\
& \operatorname{det}\left(M_{8}\right)=3 p_{1} q_{1}^{4} p_{2} q_{2}^{4} p_{3}^{2} q_{3}^{4} r_{3} \Delta^{5}>0, \\
& \operatorname{det}(M)=p_{1} q_{1}^{2} p_{2} q_{2}^{2} p_{3} q_{3}^{2} \Delta^{4}>0 .
\end{aligned}
$$

Remember that a matrix $M$ is called $P$-matrix if the determinant of every principal submatrix of $M$ is positive (see Murty [9] and Cottle et al. [10]). The class of $P$ matrices generalizes many important classes of matrices, such as positive definite matrices, $M$-matrices, and inverse $M$ matrices, and arises in applications. Note that each symmetric positive definite matrix is $P$-matrix, but the reverse is not always true. Since the matrix $M$ of our problem is $P$-matrix, we can conclude that the linear complementarity problem $\operatorname{LCP}(M, b)$ admits one and only one solution. This solution is given by

$$
\begin{aligned}
& E^{1}=\frac{1}{3} A^{-1}\left(X^{*}-\frac{c^{1}}{p q}\right) \\
& E^{2}=\frac{1}{3} A^{-1}\left(X^{*}-\frac{c^{2}}{p q}\right) .
\end{aligned}
$$

Then, the fishing effort that maximizes the profit of the first fisherman for caching the prey population is

$$
\begin{aligned}
E_{11} & =\frac{1}{3}\left[\frac{r_{1}}{q_{1}}\left(x^{*}-\frac{c_{1}}{p_{1} q_{1}}\right)+\frac{\alpha}{q_{1}}\left(y^{*}-\frac{c_{1}}{p_{2} q_{2}}\right)\right. \\
& \left.+\frac{\beta}{q_{1}}\left(z^{*}-\frac{c_{1}}{p_{3} q_{3}}\right)\right] ;
\end{aligned}
$$

the fishing effort that maximizes the profit of the first fisherman for caching the middle predator population is

$$
\begin{aligned}
E_{12} & =\frac{1}{3}\left[\frac{r_{2}}{q_{2}}\left(y^{*}-\frac{c_{1}}{p_{2} q_{2}}\right)-\frac{\bar{\alpha}}{q_{2}}\left(x^{*}-\frac{c_{1}}{p_{1} q_{1}}\right)\right. \\
& \left.+\frac{\delta}{q_{2}}\left(z^{*}-\frac{c_{1}}{p_{3} q_{3}}\right)\right] ;
\end{aligned}
$$


TABLE 2: Economic parameters of the model.

\begin{tabular}{lcc}
\hline Prey & Middle predator & Top predator \\
\hline$p_{1}=1$ & $p_{2}=3$ & $p_{3}=5$ \\
$q_{1}=0,05$ & $q_{2}=0,02$ & $q_{3}=0,01$ \\
$c_{1}=0,01$ & $c_{1}=0,01$ & $c_{1}=0,01$ \\
$c_{2}=0,015$ & $c_{2}=0,015$ & $c_{2}=0,015$ \\
\hline
\end{tabular}

the fishing effort that maximizes the profit of the first fisherman for caching the top predator population is

$$
\begin{aligned}
E_{13} & =\frac{1}{3}\left[\frac{r_{3}}{q_{3}}\left(z^{*}-\frac{c_{1}}{p_{3} q_{3}}\right)-\frac{\bar{\beta}}{q_{3}}\left(x^{*}-\frac{c_{1}}{p_{1} q_{1}}\right)\right. \\
& \left.-\frac{\bar{\delta}}{q_{3}}\left(y^{*}-\frac{c_{1}}{p_{2} q_{2}}\right)\right] .
\end{aligned}
$$

Then, the fishing effort that maximizes the profit of the second fisherman for caching the prey population is

$$
\begin{aligned}
E_{21} & =\frac{1}{3}\left[\frac{r_{1}}{q_{1}}\left(x^{*}-\frac{c_{2}}{p_{1} q_{1}}\right)+\frac{\alpha}{q_{1}}\left(y^{*}-\frac{c_{2}}{p_{2} q_{2}}\right)\right. \\
& \left.+\frac{\beta}{q_{1}}\left(z^{*}-\frac{c_{2}}{p_{3} q_{3}}\right)\right] ;
\end{aligned}
$$

the fishing effort that maximizes the profit of the second fisherman for caching the middle predator population is

$$
\begin{aligned}
E_{22} & =\frac{1}{3}\left[\frac{r_{2}}{q_{2}}\left(y^{*}-\frac{c_{2}}{p_{2} q_{2}}\right)-\frac{\bar{\alpha}}{q_{2}}\left(x^{*}-\frac{c_{2}}{p_{1} q_{1}}\right)\right. \\
& \left.+\frac{\delta}{q_{2}}\left(z^{*}-\frac{c_{2}}{p_{3} q_{3}}\right)\right] ;
\end{aligned}
$$

the fishing effort that maximizes the profit of the second fisherman for caching the top predator population is

$$
\begin{aligned}
E_{23} & =\frac{1}{3}\left[\frac{r_{3}}{q_{3}}\left(z^{*}-\frac{c_{2}}{p_{3} q_{3}}\right)-\frac{\bar{\beta}}{q_{3}}\left(x^{*}-\frac{c_{2}}{p_{1} q_{1}}\right)\right. \\
& \left.-\frac{\bar{\delta}}{q_{3}}\left(y^{*}-\frac{c_{2}}{p_{2} q_{2}}\right)\right] .
\end{aligned}
$$

\section{Numerical Simulations}

In this section, we complement the mathematical study undertaken previously on the model by numerical simulations in order to discover the effect of the variation of the price on the profits of the fishermen. The parameters of model system (1) are considered as shown in Table 1. Table 2 .

The economic parameters are considered as shown in

Using the parameters cited in Tables 1 and 2, thereafter we will see how changes in the price can affect effort fishing, catches, and profits.
TABLE 3: The influence of the price on the fishing effort.

\begin{tabular}{lcccc}
\hline$p_{1}$ & $p_{2}$ & $p_{3}$ & $E_{1}$ & $E_{2}$ \\
\hline 0,5 & 0,75 & 1 & 182,2202 & 173,3303 \\
5 & 7,5 & 10 & 198,2224 & 197,3330 \\
20 & 30 & 60 & 199,4130 & 199,1188 \\
50 & 70 & 100 & 199,7626 & 199,6433 \\
90 & 120 & 250 & 199,8282 & 199,7416 \\
220 & 400 & 510 & 199,9113 & 199,8662 \\
2200 & 3000 & 5100 & 199,9113 & 199,9915 \\
20000 & 30000 & 60000 & 199,9113 & 199,9915 \\
\hline
\end{tabular}

TABLE 4: The influence of the price on the catches.

\begin{tabular}{lcccc}
\hline$p_{1}$ & $p_{2}$ & $p_{3}$ & $H_{1}$ & $H_{2}$ \\
\hline 0,5 & 0,75 & 1 & 1,52469 & 1,38889 \\
5 & 7,5 & 10 & 1,36024 & 1,35055 \\
20 & 30 & 60 & 1,34079 & 1,33824 \\
50 & 70 & 100 & 1,33632 & 1,33531 \\
90 & 120 & 250 & 1,33554 & 1,33479 \\
220 & 400 & 510 & 1,33446 & 1,33408 \\
2200 & 3000 & 5100 & 1,33344 & 1,33340 \\
20000 & 30000 & 60000 & 1,33344 & 1,33340 \\
\hline
\end{tabular}

TABLE 5: The influence of the price on the profits.

\begin{tabular}{lcccc}
\hline$p_{1}$ & $p_{2}$ & $p_{3}$ & $\pi_{1}$ & $\pi_{2}$ \\
\hline 0,5 & 0,75 & 1 & 2 & 1 \\
5 & 7,5 & 10 & 34 & 32 \\
20 & 30 & 60 & 96 & 95 \\
50 & 70 & 100 & 229 & 228 \\
90 & 120 & 250 & 302 & 301 \\
220 & 400 & 510 & 611 & 610 \\
2200 & 3000 & 5100 & 6131 & 6130 \\
20000 & 30000 & 60000 & 71331 & 71300 \\
\hline
\end{tabular}

According to Table 3, one can remark that an increase in the price level of the three fish populations leads to an increase in the fishing effort which must be provided by each fishermen to exploit them. But on arriving at a certain rank the fishing effort becomes constant. More precisely, if the price is greater than 10300, the fishing effort in this case becomes constant and it does not exceed 399,9781. This means that the fishermen must not exceed a fishing effort equal to 399,9781 to expect maximum benefit by making more reasonable catches that take into account the preservation of marine species.

According to Table 4, the catch level decreases as the price increases. If the price is equal to 4000 , the catches that maximize the profit of the fishermen are equal to 2,66684; in this case the catches that allow the fisherman to have a maximum profit do not exceed 2,66684. Contrariwise, the profit is always increased even if the level of catches decreases, which is justified by the increase in the level price of fish populations (Table 5). 
Consequently, it can be deduced that this model meets the objective of the work since it allows the fishermen to maximize their profit taking into account the preservation of marine recourses.

\section{Conclusion}

In the present paper, we have studied a bioeconomic tritrophic prey-predator model. We have maximized the profit of two fishermen exploiting the prey, middle predator, and top predator fish populations. The existence and stability of equilibrium point are studied using eigenvalue analysis and Routh-Hurwitz criterion. Using the Nash equilibrium problem and linear complementarity problem we have determined the equilibrium point that maximizes the profits of each fisherman. We have closed this paper by some numerical simulations in order to show the influence of the price on the profits of fishermen.

\section{Conflicts of Interest}

The authors declare that there are no conflicts of interest regarding the publication of this paper.

\section{References}

[1] J. H. Dawes and M. O. Souza, "A derivation of Holling's type I, II and III functional responses in predator-prey systems," Journal of Theoretical Biology, vol. 327, pp. 11-22, 2013.

[2] Y. Lu, K. A. Pawelek, and S. Liu, "A stage-structured predatorprey model with predation over juvenile prey," Applied Mathematics and Computation, vol. 297, pp. 115-130, 2017.

[3] Y. Qi and Y. Zhu, "The study of global stability of a diffusive Holling-Tanner predator-prey model," Applied Mathematics Letters, vol. 57, pp. 132-138, 2016.

[4] W. Mbava, J. Y. Mugisha, and J. W. Gonsalves, "Prey, predator and super-predator model with disease in the super-predator," Applied Mathematics and Computation, vol. 297, pp. 92-114, 2017.

[5] P. Santra, G. S. Mahapatra, and D. Pal, "Analysis of differentialalgebraic prey-predator dynamical model with super predator harvesting on economic perspective," International Journal of Dynamics and Control, vol. 4, no. 3, pp. 266-274, 2016.

[6] A. Ghorai and T. K. Kar, "Biological control of a predatorprey system through provision of a super predator," Nonlinear Dynamics, vol. 74, no. 4, pp. 1029-1040, 2013.

[7] K. Chouayakh, C. EL Bekkali, Y. EL Foutayeni, M. Khaladi, and M. Rachik, "Maximization of the Fishermen's Profits Exploiting a Fish Population in Several Fishery Zones," International Journal of Science and Research, pp. 2319-7064, 2013.

[8] R. P. Gupta and P. Chandra, "Dynamical properties of a preypredator-scavenger model with quadratic harvesting," Communications in Nonlinear Science and Numerical Simulation, vol. 49, pp. 202-214, 2017.

[9] K. G. Murty, "On the number of solutions to the complementarity problem and spanning properties of complementary cones," Linear Algebra and its Applications, vol. 5, no. 1, pp. 65-108, 1972.

[10] R. W. Cottle, J.-S. Pang, and R. E. Stone, The Linear Complementarity Problem, Academic Press, Boston, Mass, USA, 1992. 


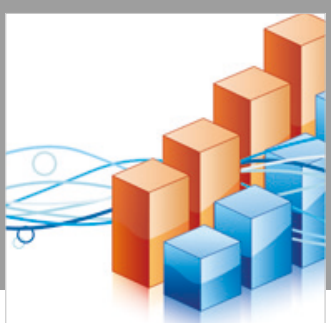

Advances in

Operations Research

\section{-n-m}
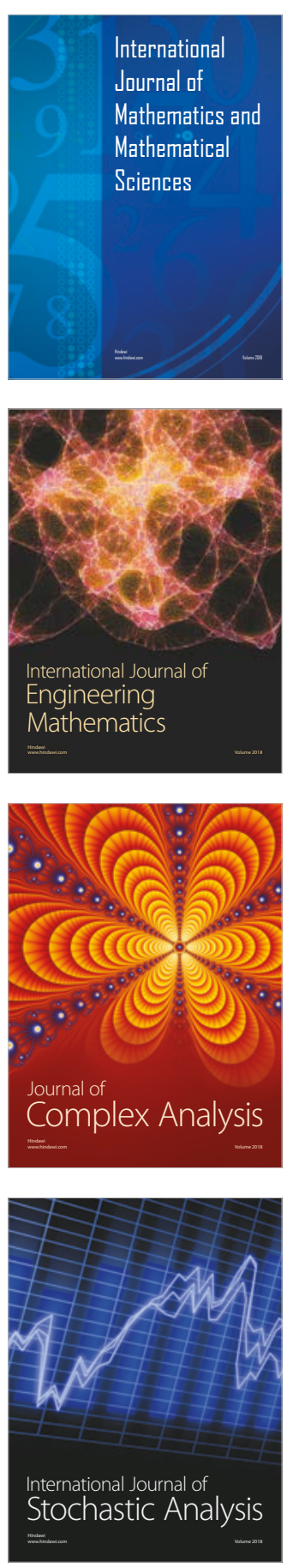
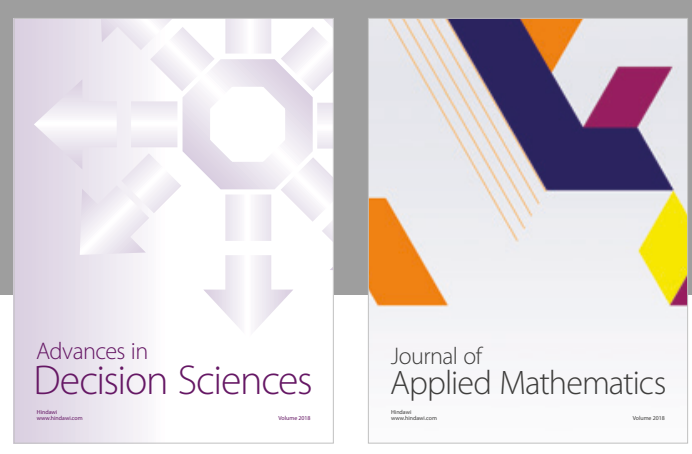

Journal of

Applied Mathematics
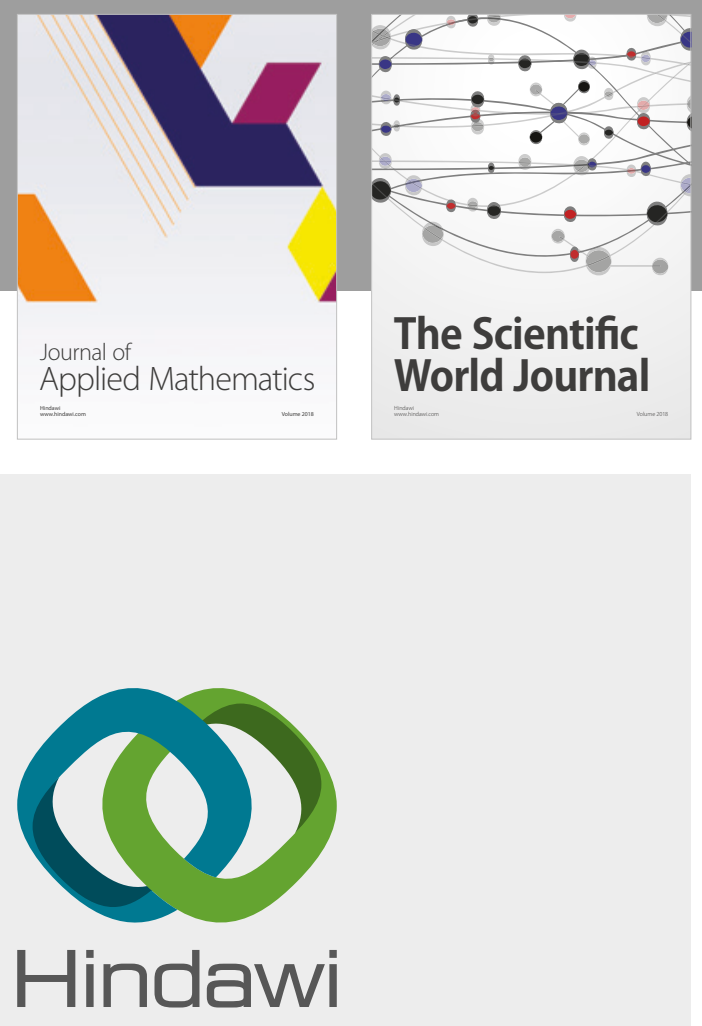

Submit your manuscripts at

www.hindawi.com

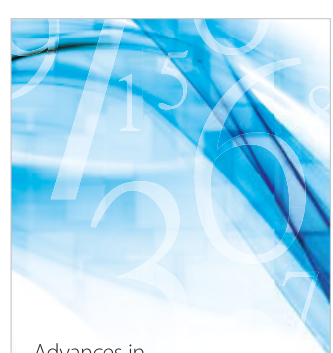

Advances in
Numerical Analysis
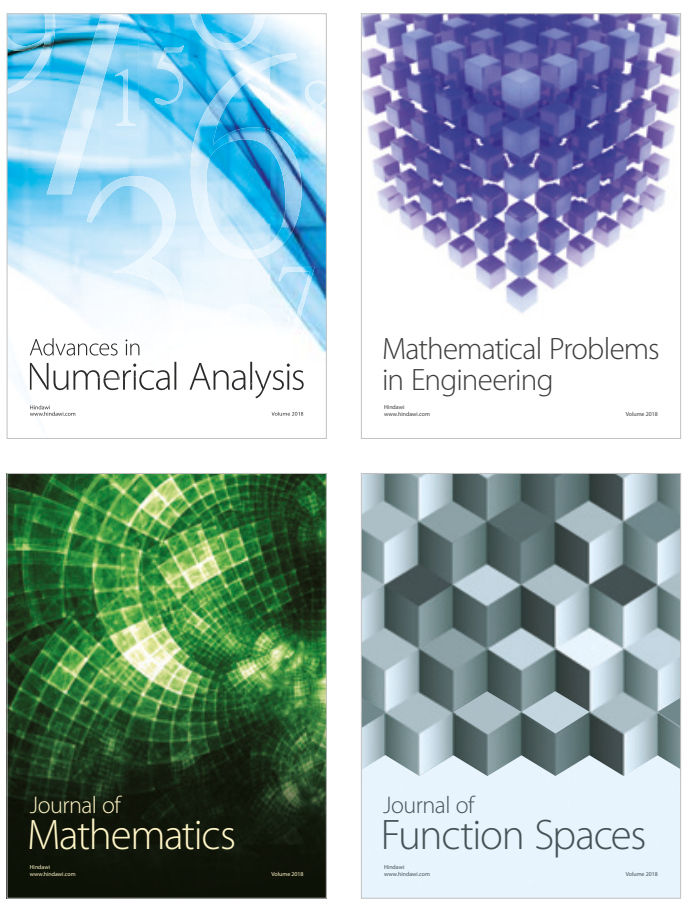

Mathematical Problems in Engineering

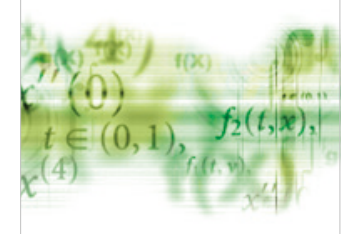

International Journal of

Differential Equations

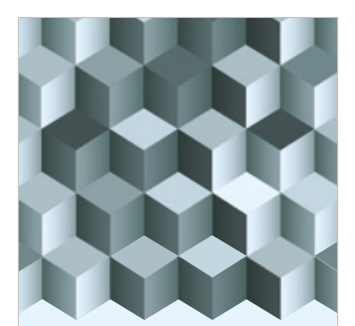

Journal of

Function Spaces

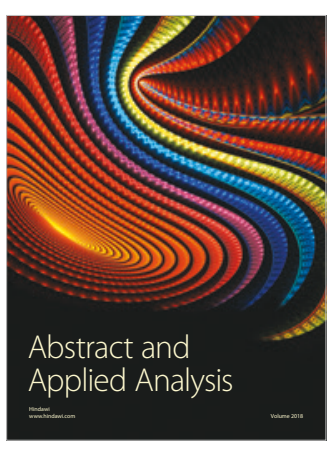

The Scientific

World Journal

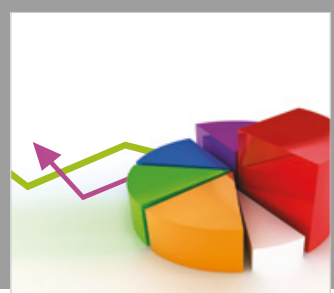

Journal of

Probability and Statistics
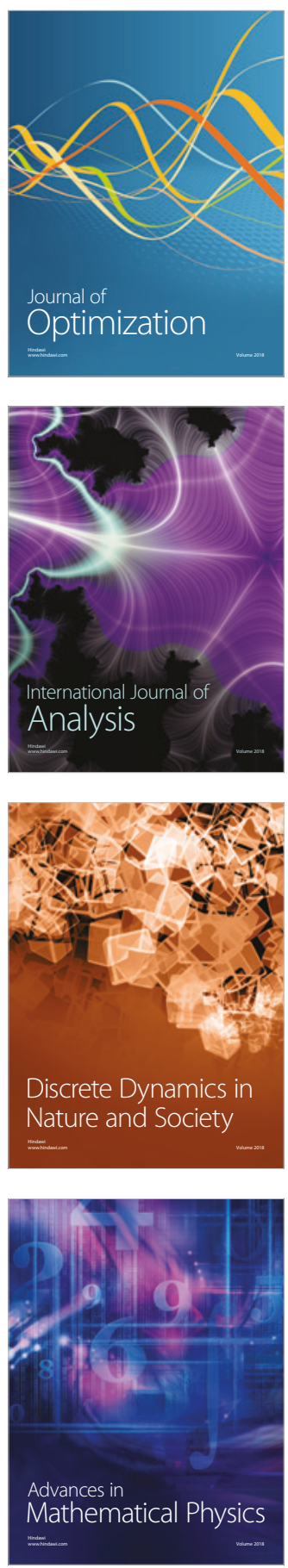